\title{
MEANING, PROTOTYPES AND THE FUTURE OF COGNITIVE SCIENCE
}

\author{
J. van Brakel ${ }^{1}$
}

One consequence of cognitive science's take-over-bid for a naturalised philosophy is that empirical psychological theories are taken more and more seriously by philosophers. In this paper I'll discuss one current empirical paradigm, viz. prototype theory. Prototypes would equip us with foundations in practice, because all human cognition would be governed by the same epistemological and/or mental and/or semantic primitives, viz. PROTOTYPES.

First I'll explain what prototypes are, followed by examples of recent appeals to prototypes in the philosophical literature. Then I'll evaluate the soundness of the prototype paradigm, in particular its core assumption that prototypes provide some sort of universal psychological essences (at least at this point in evolution), explaining why, on the whole, human communication and learning are successful.

\section{@1 what prototypes are}

Since its inception by Rosch, the concept of prototype has had an enormous impact in cognitive science. ${ }^{2}$ Combined with the idea of basic level categories, it is representative of the cluster of

\footnotetext{
${ }^{1}$ Department of Philosophy, University of Utrecht, P.O. Box 80.103, 3584 TC Utrecht, Netherlands. This paper was written while on leave at the Department of Philosophy of Simon Fraser University, Burnaby, B.C., Canada.

${ }^{2}$ In the Citation Index there have already been more than 2000 citations of Rosch's work on prototypes. For an overview see C. Mervis and E. Rosch, Categorization of natural objects, Annual Review of Psychology, 32 (1981) 89-115; E. Smith and D. Medin, Categories and Concepts, Cambridge MA 1981; or G. Lakoff, Women, Fire and Dangerous Things, Chicago 1987 (see also references in note 16). Recent publications from various fields in which the concept of prototype is discussed or used include: S. Atran, Ordinary constraints on the semantics of living kinds, Mind \& Language, 2 (1987) 27-63; E.R. MacCormac, A Cognitive Theory of Metaphor, Cambridge MA 1985; C. Craig (ed.), Noun Classes and Categorization, Amsterdam 1986; S. Harnad (ed.) Categorical Perception, Cambridge 1987; D. Hilton (ed.), Contemporary Science and Natural Explanation, Sussex 1988; U. Neisser (ed.), Concepts and Conceptual Development, Cambridge 1987; B. Rudzaka-Ostyn (ed.), Topics in Cognitive Linguistics, Amsterdam 1988; R. Jackendoff, Semantics and Cognition, Cambridge MA 1983; and R. Jackendoff, Consciousness and the Computational Mind, Cambridge MA 1987. See also references in notes 5, 8, 9, 13-15.
} 
concepts that have been invented to deal with the frame problem in Artificial Intelligence. ${ }^{1}$ Basic-level categories are functionally, epistemologically, and linguistically primary or most salient. They are determined by experiential aspects of human psychology such as gestalt perception (which picks out the objects having roughly the same shape) and interactional clusters of everyday human motor activities. The words for basic-level categories are learnt first and introduced ostensively; they are short, most often used by speakers of the language and have greater cultural significance than other words. In short: They name the foundational categories to get off the ground in getting to know the world and talk about it to others. ${ }^{2}$ As Fodor writes about emotions: ${ }^{3}$

Consider such folk-psychological concepts as ANGRY, SAD, HAPPY, etc. I think there's no doubt that these are acquired early, that they must have been part of the universal prehistory of our species, and that they are easily introduced by ostension.

According to prototype theory, all basic level categories display prototype effects and in the sequel I'll use "prototype" as short-hand for "basic-level categories with prototype structure". Prototypical exemplars form the core or centre, while the boundaries of the category are fuzzy. For example, there are considerable differences in what people in different cultures, or even in one culture, include under "green", but, according to prototype theory, they will all agree (approximately) on one particular focal or unique green, which is the most salient pan-human prototypical exemplar of the category.

\footnotetext{
${ }^{1}$ The frame problem refers to the problem of establishing the facts that may be relevant to a certain action, including the facts that will not change as a result of the action. See Z.W. Pylyshyn (ed.), The Robot's Dilemma, Norwood NJ 1987.

${ }^{2}$ But they are not necessarily natural kinds in the Kripke/Putnam sense (contrary to what is suggested in many publications). Stereotypes are different from prototypes because the former are descriptions stated in words, while the latter aim at something deeper than words. [H. Putnam, Representation and Reality, Cambridge MA 1988:44.]

${ }^{3}$ J. Fodor, Representations, Cambridge MA 1981:312.
} 
Examples of such basic prototypes include: classifications of plants and animals at the genus level, e.g. Western Red Cedar, tigre; basic colour terms: red, green, yellow, blue; basic emotion modes such as happiness or anger; basic tools: pot, hammer, etc.; and basic spatial metaphors like in/out and path/goal.

\section{@2 appeal to prototypes}

The earliest philosophical appeals to prototype theory aim at providing the content of modules, which process environmental input via wired-in basic categories into semantic universals. This idea has been worked out in quite some detail for colour. For example, Hardin, in his $1988 \underline{\text { Color }}$ for Philosophers, writes: ${ }^{1}$

The Berlin and Kay basic color categories are simply the product of a set of filters at an early stage of neural processing [167]... the basic linguistic categories, as shown by Berlin and Kay to be apparent in all languages, themselves have been induced by the existence of a natural, biologically induced set of hue categories, whose perceptual saliences leave their traces in all natural languages [168]. [Hence] biology determines phenomenology and, in consequence, a piece of semantic structure. [156]

Similarly, with respect to emotion terms, Lakoff (1987) reports that Ekman's seven prototypical emotions are readily recognisable by gestalt perception around the world and correspond to particular activities in the autonomic nervous system. ${ }^{2}$ And Fodor talks about "a natural

\footnotetext{
${ }^{1}$ For a criticism of this view see J. van Brakel, The plasticity of categories: the case of colour, Brit. J. Phil. Sci., in press.

${ }^{2}$ Competing theories exist based on six, seven, eight, nine, ten, or eleven basic emotions. The most recent 1989 study of Johnson-Laird and Oatley claims that there are exactly five basic emotion modes: P.N. Johnson-Laird and K. Oatley, The language of emotions, Cognition and Emotion, 3 (1989) 81-123. See further contributions in K.R. Scherer and P. Ekman, Approaches to Emotion, Hillsdale NJ 1984; C.E. Izard et al, Emotions, Cognitions and Behavior, Cambridge. Confusion about the number of basic emotion terms and their relation to other terms is often embarrassing. For example Lakoff 1987:38 writes: "The seven basic emotions appear to have prototype states." But the only emotions he mentions, referring to the work of Ekman on pan-cultural facial expressions, are happiness, anger, fear, surprise, interest, and sadness (hence six emotions). In one of his most recent publications Ekman also lists six basic emotions, but Lakoff's interest and sadness are replaced by disgust and distress. It's also interesting to note that Lakoff's basic emotion "interest" doesn't even occur in Johnson-Laird and Oatley's list of 595 emotion
} 
ontogenetic bias in favor of [such] concepts". ${ }^{1}$ As a consequence, prototype terminology pervades discussions in cognitive science about frames and schemata and is invoked by philosophers defending RATIONALITY against "the relativists".

More recently, the prototype paradigm has also been embraced in connectionist circles. Here prototypical patterns, representing concepts or specific knowledge, are associated with activation-vector spaces at the hidden level of the connectionist network. ${ }^{2}$ In fact, as Churchland and others have argued, prototypes go more naturally with probabilistic networks, while GOFAI (Good Old Fashioned Intelligence) fits better with strictly definitional entities. Even where there is no reference to Rosch or prototypes, the paradigm is omnipresent. For example, in a recent issue of the journal $\underline{\text { Cognitive Science, Hadley writes: }}{ }^{3}$

An object which is recognized by a PDP net as instantiating a particular concept will possess a salient subset of the properties in the CORE set.

\section{An Clark comments on a similar PDP-net: ${ }^{4}$}

Plato scholars will envy the system's ability to see the true form of doghood on the basis of these distorted shadows on the wall of the cave.

words. One problem is of course that not all writers agree on how to separate emotions from such things as reflexes, moods, emotional traits, and emotional disorders, nor do they agree on the "amount" of cognitive, affective, intentional, and moral factors in emotions and their relation to changes in the autonomic and central nervous system.

${ }^{1}$ Fodor 1981:311. "So, the suggestion is that the visual-input system delivers basic categorizations." [J.A. Fodor, The Modularity of Mind, Cambridge MA 1983:97.] Cf also J.A. Fodor, Psychosemantics, Cambridge MA 1987:114-122.

2 See P.M. Churchland, Matter and Consciousness, Cambridge MA 1988:161; P.M. Churchland, A Neurocomputational Perspective, Cambridge MA 1989:122f,209-223; J.L. McClelland and D.E. Rumelhart, A distributed model of human learning and memory, in J.L. McClelland et al, Parallel Distributed Processing, vol. 2, Cambridge MA 1986; W. Bechtel, Connectionism and the philosophy of mind, The Southern Journal of Philosophy, 26 (1987) Supplement.

${ }^{3}$ R.F. Hadley, A default-oriented theory of procedural semantics, Cognitive Science, 13 (1989) 107-137.

${ }^{4}$ A. Clark, Microcognition, Cambridge MA 1989:99. In fairness it should be added that the network was never exposed to an exemplar of the prototype, only to distorted instances. 
but I would guess that Plato scholars will more envy the simplicity of the systems' Platonic world, in which the researcher has fixed the prototypical dog in terms of exactly sixteen features. PDP-systems may be good at picking up cues in their teaching input, but it is the teacher's prototypes that are represented, not those of the systems' or the environment's making. ${ }^{1}$

Further, prototypes are referred to in philosophical arguments by many influential philosophers; for example Burge, Churchland, Fodor, Goldman, Lycan, Kornblith, Ruse, Stich, and Schiffer. ${ }^{2}$ Typically Stich writes:

The notion of prototype has received considerable attention in recent empirical work on the mental representation of concepts, and it will play a prominent role in my account of belief.

Most philosophers referring to prototypes take the western culture as the only cognitive reference point. Although reference is made to the human mind, in fact discussions of examples are restricted to the Western Mind, if not the English Speaking Mind. But of course, if there are such things as prototypes, which correspond to linguistic universals, epistemological primitives, or innate mental schemata, they have to exist for all cultures.

\section{@3 empirical criticisms of prototype theory}

\footnotetext{
${ }^{1}$ In fact, because of the more explicit role of the teacher, PDP-models are easier to converge with a Wittgensteinian account then GOFAI-models; see for example G. Gillett, Representation and cognitive science, Inquiry, 32 (1989) 261-276.

${ }^{2}$ See: S. Stich, From Folk Psychology to Cognitive Science, Cambridge MA 1989:249; W.G. Lycan, Tacit belief, in R.J. Bogdan, Belief, Oxford 1986:72; also in his Judgement and Justification, Cambridge 1988:64; A.I. Goldman, Interpretation psychologized, Mind \& Language, 4 (1989) 161-185; MacCormac 1985:75; several contributions on "What is a concept?" in a special issue of Mind and Language, 4 (1989) Nos 1 and 2; T. Burge, Individualism and psychology, The Philosophical Review, 95 (1986) 6; H. Kornblith, Naturalistic epistemology, lecture 1990 APA Pacific Div. Mtg.; C. Cherniak, Minimal Rationality, Cambridge MA 1986:89; W. de Pater and W. van Langendonck, Natuurlijkheid van de taal en iconiciteit, Tijdschrift voor Filosofie, 51 (1989) 256-297; M. Ruse and E.O. Wilson, Moral philosophy as applied science, Philosophy, 61 (1986) 173-192; Th.W. Schick, The semantic role of qualitative content, The Southern Journal of Philosophy, 27 (1989) 125-133; S. Schiffer, The real trouble with propositions, in Bogdan 1986; A.I. Goldman, Cognition and Content, Cambridge MA 1986:321f. See also references in notes 5, 8, and 9.
} 
As it happens, recent evidence suggests that our judgments of prototypicality are contextdependent and generally not as stable as Rosch's initial data suggested. Instead, recent alternative approaches in psychology emphasise that categories are open-ended and stress the importance of theory-driven categorization, concept modifications associated with context effects, and the dynamic restructuring of information. ${ }^{1}$

Of course the latter views don't prove prototype theory is wrong, but it does show that if philosophers let empirical theories in, they should give up the idea that such theories are prototypical rockbottoms to support philosophical positions.

\section{@4 prototypes and Wittgenstein}

It is now almost canonical to refer to Wittgenstein and Rosch in the same breath, when prototype theory is introduced. ${ }^{2}$ However, it is not at all clear what exactly the resemblance is between the views of Wittgenstein and Rosch. With the notion of 'family resemblance' Wittgenstein wanted to illustrate that there is no uniform set of attributes which constitutes the meaning of a linguistic

\footnotetext{
${ }^{1}$ For example Wattenmaker et al conclude that "the probabilistic [=prototype] view is fatally flawed" and Neisser, summarising recent work says: "Typicality, it seems, is typically quite variable." See W.D. Wattenmaker, G.V. Nakamura and D.L. Medin, Relationships between similarity-based and explanation-based categorization, in Hilton 1988:204-240; U. Neisser, From direct perception to conceptual structure, in Neisser 1987:11-24. See further: F.C. Keil, Conceptual development and category structure, in Neisser 1987:175-200; D.L. Medin and E.J. Shoben, Context and structure in conceptual combination, Cognitive Psychology, 20 (1988) 158-190; S.A. Gelman, The development of induction within natural kind and artifact categories, Cognitive Psychology, 20 (1988) 65-95. Cf also S. Carey, Conceptual Change in Childhood, Cambridge MA 1985; E. Roth and E. Shoben, The effect of context on the structure of categories, Cognitive Psychology, 15 (1983) 346-378; L. Barsalou, The instability of graded structures: Implications for the nature of concepts, in Neisser 1987.

Earlier criticisms include: If prototypes are representations it's not clear how the basic categories can be extended to complex categories. Concepts which have exact definitions (odd number, rectangle, grandmother, etc.) also display prototype effects. The concept is of little use in structuring concepts like verbs. The first widely quoted criticism was probably that of S.L.Armstrong, L.R. Gleitman and H. Gleitman [What some concepts might not be, Cognition, 13 (1983) 263-308], but it has been dismissed by fierce cognitivists like Jackendoff (1987:143; 1983:255) and Lakoff (1987:148-152).

${ }^{2}$ Recent references to Wittgenstein and Rosch's prototype theory include: S.P. Stich, Are belief predicates systematically ambiguous, in R.J. Bogdan (ed.), Belief, Oxford 1986; P. Churchland, lecture 1989 APA Pacific Division Meeting; G.M. Edelman, Neural Darwinism, New York 1987; T. Givon, Prototypes: Between Plato and Wittgenstein, in Craig 1986; D. Geeraerts, Where does prototypicality come from?, in Rudzaka-Ostyn 1988; R.M. French, Subcognition and the limits of the Turing test, Mind, 99 (1990) 53-65; J.R. Taylor, Linguistic Categorization, Oxford 1989:38-55; P. Thagard, Computational Philosophy of Science, Bradford 1988:18; K.R. Livingston, Concepts, categories, and epistemology, Philosophia, 19 (1989) 265-300; N.A. Stillings et al, Cognitive Science, Cambridge MA 1987:66; Atran 1987; Jackendoff 1987.
} 
expression. There are only similarities between different uses of an expression. However, in Rosch's publications we find reference to "Platonic forms", "ideal types", entities "determining categorical structure", "a measure of family resemblance", and "the center of a 'Wittgensteinian' category" [emphasis added], ${ }^{1}$ which expressions seem to have little application to the views of the later Wittgenstein. ${ }^{2}$

\section{@ 5 questions to be asked}

The force of prototype theory is that, in Lakatosian fashion, one can keep the core fixed while allowing lots of variation at the periphery. It's clear that if one starts with a set of epistemological primitives which have an absolutely fixed core, but a fuzzy surround, plus recursive rules, which are also a little bit fuzzy at the edges of application, one can explain any variation in actual data, and still have a rock-bottom for philosophical justification.

The question is not, of course, whether there is anything at all which people all over the world share. ${ }^{3}$ To consider this question seriously would make it sheer magic that when Cook arrived at Nootka sound in March 1778, making the first white contact with the Nuu-chah-nulth people of the village of Yuquot on Vancouver Island, the following events happened: ${ }^{4}$

\footnotetext{
${ }^{1}$ Quotations from: E. Rosch, Linguistic relativity, in: Human Communication: Theoretical Explorations, New York 1974:114; E. Rosch, Cognitive reference points, Cognitive Psychology, 7 (1975) 532-547; E. Rosch and C. Mervis, Family resemblances, Cognitive Psychology, 7 (1975) 573-605; E. Rosch, Wittgenstein and categorization research in cognitive psychology, in Meaning and the Growth of Understanding (M. Chapman \& R.A. Dixon, eds.), Berlin 1987; E. Rosch, Coherences and categorization, in F. Kessel (ed.), The Development of Language and Language Researchers, Lawrence Erlbaum 1988:373-392.

${ }^{2}$ There is also an ambiguity as to whether prototypes correspond to salient features of the world out there, to physiologically grounded mental codes, or to concepts in some abstract domain. This comes across clearly in the variety of criticisms that have been levelled at prototype theory. Some writers have criticised it for the mistaken assumption that one could have a prototypical tiger in one's head; others have criticised it for focusing on the structure of the environment at the cost of neglecting the nature of the organism which develops and uses concepts.

${ }^{3}$ The situation is not that I first recognise my common humanity with others and that this recognition then provides the intellectual justification for my response to certain modalities in my dealings with them. On the contrary, the recognition itself is a function of those responses: "our unreflective reactions are part of the primitive material out of which our concept of a human person is formed and which makes (such) more sophisticated reflections possible". [P. Winch, Trying To Make Sense, Oxford 1987:147.]

${ }^{4}$ Quoted from Samwell's journal: The Voyage of the Resolution and Discovery 1776-1780, J.C. Beaglehole (ed.), part 2, Cambridge: University Press, 1967:1088. Cook's ship was not the first European contact with Vancouver Island. In 1774 the ship of Juan P*rez made contact with people from the village of Hesquiat. Probably this news also got to the village of Yuquot. There are many similarities between the accounts of these two first contacts:
} 
As we were coming [in] we were surrounded by thirty or 40 Canoes full of Indians who expressed much astonishment at seeing the Ship; they stood up in their Canoes, made many strange Motions, sometimes pointing to the shore $\&$ at other times speaking to us in a confused Manner very loud $\&$ shouting, \& presently after they all sung in concert in a wild Manner, ... We made Signs of Friendship to them and invited them along side the Ship where they soon ventured \& behaved in a peaceable manner, offering us their Cloaths \& other things they had in their Canoes, and trading immediately commenced between us; ... Night coming on they all paddled ashore except five or six Canoes which drew in a Cluster together at a small distance from the Ship, and as it were to bid us a good night the people in them sang in concert in no disagreeable Stile; this Mark of their Attention to us we were unwilling to pass over unnoticed \& therefore gave them in return a few tunes on two french Horns after their Song was ended, to these they were very attentive, not a word to be heard among them during the time of playing; this salutation was returned by another Song from the Indians, after which we gave them a Tune on the Drum \& Fife to which they paid the same attention as they had done to the Horns.

There's no need to worry whether, theoretically speaking, communication between human beings might be impossible, but it doesn't follow that therefore there's a rockbottom of shared "primitive" prototypes. The questions to be asked are:

1. How hard is the core of a prototype? Perhaps any part of the periphery can move in the place of the core.

2. How many cores are there? Perhaps there are many different ways of asking what the cores are.

\footnotetext{
About eight o'clock at night three canoes ... came to us; but they remained at a distance from the ship, their occupants crying out in a mournful tone of voice. We called to them, and they drew near. Shortly afterward they said goodbye, but, until after eleven o'clock, they remained at a distance of about a musket-shot from the ship, talking among themselves and sometimes crying out. ... [the next day] there arrived fifteen canoes ... We gave them to understand that they might draw near without fear, and presently they came to us and began to trade with our people.
}

[From the diary of Fray Tomas de la Pe:a, in D.C. Cutter, The California Coast, Norman OH, 1969, pp. 178-183.] 
3. How much follows from a particular family of cores in terms of predicting human behaviour? Perhaps nothing very definitive.

These questions undermine two major assumptions of prototype theory, cognitive science, and naturalised philosophy:

Firstly, the assumption that everything said is to fit some cognitive model or other; the assumption that cognition is just a quantity of stuff for which the scientific laws giving its statics and dynamics need be found; the assumption that all practices of using words can be explained, while overlooking the fact that any such explanations presuppose practices. ${ }^{1}$

Secondly, the assumption that there are a fixed number of cognitive domains, each containing a fixed number of invariant prototypical categories. On such a view, all domains together make up one closed system, one cognitive space which embodies intelligent thinking. To find out how human organisms think (i.e. to find how their cognitive space is organised), they are, as it were, being taken apart (as in interviewing specialists by knowledge engineers or in emic measurements by ethnopsychologists). The closedness of this approach can be contrasted with, for example, Wittgensteinian forms of life. ${ }^{2}$

In further elucidating the provisional answers to the three questions just mentioned, I'll consider a number of ways in which the prototype concept is not a Wittgensteinian type of family resemblance. My point here is not to give the better exegesis of Wittgenstein. ${ }^{3}$ I've chosen $^{\prime}$ mainly Wittgensteinian terminology, because his name has been used to give philosophical

\footnotetext{
${ }^{1}$ Some practices are shared and understood, although (ultimate, scientific) explanations or grounds for them cannot be given. (Grunde gibt es erst in einem Sprachspiel [Wittgenstein, Bem.Phil.Psych. 2,689].)

${ }^{2}$ Compare Margolis' definition of forms of life: "the ultimately unsystematizable complex of actual societal life on which any provisionally formulable regularities or rules of behaviour are based" [Wittgenstein's "Forms of Life", in Meaning and the Growth of Understanding (M. Chapman \& R.A. Dixon, eds.), Berlin 1987:132. Cf. Wittgenstein, PI:240-242, 223e-226e.

${ }^{3}$ See for Wittgensteinian critiques of cognitive science for example contributions of J. Coulter, J. Margolis and J. Russell in Meaning and the Growth of Understanding (M. Chapman \& R.A. Dixon, eds.), Berlin: Springer, 1987; P. Hacker, Languages, minds and brains, in Mind Waves (C. Blakemore \& S. Greenfield, eds.), Oxford 1987: 485-505; J. Coulter, Rethinking Cognitive Theory, London 1983; R. Harr*, Wittgenstein and artificial intelligence, Philosophy and Psychology, 1 (1988) 105-115; O. Neumaier, A Wittgensteinian view of artificial intelligence, in Artificial Intelligence: The Case Against (R. Bron, ed.), London 1987: 132-173; O. Neumaier, Mentalismus in der Cognitive Science, Z. phil. Forsch., 43 (1989) 331-346; R. McDonough, Towards a non-mechanistic theory of meaning, Mind, 98 (1989) 1-21; J. Margolis, Science Without Unity, Oxford 1987:436-449.
} 
weight to the prototype concept. Moreover, in the spirit of naturalising philosophy, I will appeal for support to empirical data and briefly discuss two examples drawn from anthropology. ${ }^{1}$

\section{@6 example 1: sweet potatoes}

Consider the Dani, who live in the New Guinea Highlands. According to Heider they have more than 70 words referring to sweet potatoes. ${ }^{2}$ Now, it's well documented that sweet potatoes are extremely important in the Dani culture. More than half of all conversations are directly or indirectly about sweet potatoes.

After having spend some time trying to find out which sweet potatoes were called by what name, Heider gave up, concluding that "although there are more than seventy terms for sweet potatoes, they are used with considerable imprecision." He also noted that the Dani explicitly denied that different types of sweet potatoes have different susceptibilities.

Of course, Heider may not have tried hard enough. But assume somebody came up with a book length account unravelling "everything" about which sweet potato words should be taken as basic and which as peripheral and explaining which cognitive models about horticulture, food preparation, etc are interrelated here via several sorts of universal metonymic or whatever relations. ${ }^{3}$ Would it really make sense to see such a result as anything more than one way to look at the Dani sweet potato world, let alone as the best Dani way to look at sweet potatoes?

Another way to look at the inconsistency of the way many of the Dani words for sweet potatoes are used--not the way to look at it--is to contemplate that also in English such

\footnotetext{
${ }^{1}$ In a longer version of this paper I also discuss examples concerning botanical classification and the universality of basic emotions.

${ }^{2}$ K.G. Heider, The Dugun Dani, Chicago 1970:32f.

${ }^{3}$ Finding such cognitive or cultural models is the current aim of cognitive anthropology (ethnoscience). A typical example is E. Hutchins, Culture and Inference: A Trobriand Case Study, Cambridge 1980. He gives a very detailed cognitive model of the Trobriand's practice of land litigation. Earlier studies had suggested that their language Kiriwana doesn't allow logical reasoning; for example Kiriwana lacks a particle equivalent to English "and". Hutchins "uncovers" the devices used in Kiriwana to accomplish conjunction and other important reasoning devices such as cause and effect constructions, by adding a large amount of contextual information to what is actually said. However, it doesn't follow therefore that the Trobriander are actually using Hutchins' model; nor does it follow from the absence of connectives in the sense we know them that the Trobriander are incapable of logical reasoning or reasoning in terms of cause and effect.
} 
inconsistencies may occur. For example, vocabulary for the description of the taste and odour of wines does not seem to have any fixed meaning, even among experts, but seems to function primarily in constructing a vague tone of knowledgeableness and concern about wine among a group of speakers. ${ }^{1}$

\section{@7 example 2: the domain of colour}

According to the theory of Berlin and Kay, there exist eleven Basic Colour Terms (BCTs), which name eleven linguistic and cognitive universals. Later additions have provided a neurophysiological grounding of the 11 BCTs and sociobiologists have grounded them further in genetics. ${ }^{2}$ There are many languages which have less than 11 BCTs, but, according to Berlin and Kay, they all fit a particular evolutionary order of BCT-development. I won't comment here on the potential racist implications of the evolutionary part of the theory and just briefly mention some of the major ethnocentric assumptions that are made to fit the data of the world's languages into one universal theory about prototypical colours.

Firstly, there's the assumption that eventually all primitives can be grounded in neurophysiology. As far as I can see, neurophysiology sets no constraints whatsoever on the categorisation of colours, ${ }^{3}$ but more telling is that the approach to ground BCTs this way is incoherent on its own terms. For example, many North-west Coast languages have one term covering both green and yellow. Much to the amazement of the author reporting this fact, it "contravenes what current knowledge of visual physiology would lead us to expect". ${ }^{4}$ Would it

\footnotetext{
${ }^{1}$ See A. Lehrer, Wine and Conversation, Bloomington 1983. This is what the anthropologist Malinowski called "phatic communion".

${ }^{2}$ See for the neurophysiological grounding: P. Kay and C.K. McDaniel, The Linguistic Significance of the Meanings of Basic Color Terms, Language, 54 (1978) 610-646; also summarised in Lakoff 1987:24-30. For the sociobiological account: C. Lumsden, Color categorization: A possible concordance between genes and culture, Proc. Natl. Acad. Sci. USA, 82 (1985) 5805-5808.

${ }^{3}$ See my The plasticity of categories: the case of colour, Brit. J. Phil. Sci., in press.

${ }^{4}$ R.E. MacLaury, Color-Category Evolution and Shuswap Yellow-with-Green, American Anthropologist, 89 (1987) 107-24. There seem to be at least as many languages which have one word for blue and green as there are which have separate words. This fact would not contradict the neurophysiology of colour vision. The "disturbing" combination of green and yellow in one category is more rare. In old Greek the term chloros roughly covers a colour space consisting of colours like those of olives, olive wood, leeks, certain branches, ripe corn, honey, certain hair,
} 
really be possible that the meaning of a word in another culture contradicts our physiological knowledge?

Secondly, there's the hidden assumption that what is salient has to be named. But the fact that we don't have Basic Smell Terms doesn't mean that the Western Flavours \& Flagrances Syndicate is underdeveloped. Similarly, the fact that there are no words for certain colours doesn't say very much about the cultural significance of the colour. In Old Egypt people managed for thousands of years without a word for blue. But painters used seven and later nine primary colours of which blue was "the most prestigious painted colour".

Thirdly, there's the obvious restriction to exactly one domain. Not only do cultures vary in the number of basic colour terms they have, it's often not clear at all whether there is a separate domain of colour. The hue aspect of colour may, as it were, be subsumed under different categories, so that colour is not really present as a separate domain; still there will be words like colour words. For example, Lokono (Surinam) has three basic colour terms covering the brightness or intensity of light: karimeto [dark/black], harrirato [light/white], subuleto [various medium bright colours: yellow, blue, pink, peach, orange, tan]. In addition it has three basic terms which are "shared" between the domains of colour and ripeness: imoroto [unripe, green], $\underline{\text { koreto }}$ [ripe, red], and bunaroto [overripe, brown/purple]. ${ }^{2}$ There are many similar examples in other cultures: The domain of colour may overlap with the domain of form, with the domain of ritual, the domain of evaluatory terms, and so on. ${ }^{3}$

some eye colours, certain white wines, withered leaves, palefacedness, the sea (sometimes), and the nightingale and might best be translated as yellow-green [A.E. Taylor, A Commentary on Plato's Timaeus, Oxford: Clarendon Press, 1964].

1 J. Baines, Color Terminology and Color Classification: Ancient Egyptian Color Terminology and Polychromy, American Anthropologist, 87 (1985) 282-297.

${ }^{2}$ N. Hickerson, A Reconsideration of Lokono Color Terms, paper presented at the Int. Congress of Americanists, Amsterdam 1988.

${ }^{3}$ For more examples and a criticism of the work of Rosch, Berlin and Kay, and Hardin on colour see C. Ratner, A sociohistorical critique of naturalistic theories of color perception, J. Mind Behav., 10 (1989) 361-372; B.A.C. Saunders and J. van Brakel, Re-evaluating Basic Colour Terms, Cultural Dynamics, 1 (1988) 359-378; On crosscultural colour semantics, Int. J. Moral and Social Studies, 4 (1989) 173-180; Against basic colour terms, Proc. Symp. Between Semantics and Rationality (12th Int. Congr. Anthropological and Ethnological Sciences, Zagreb, July 1988), in press. 
Finally, there's a strong constraint on the kind of data that are allowed to enter the discourse. For example, the distinction between decontextualised basic colours and "other" colours is introduced by definition and not open to "falsification". Consider, for example the experience of Luria with Ichkari women (from Uzbekistan), who refused to do basic colour naming experiments with coloured threads saying things like "this is like calf's dung, and this is like a peach; you can't put them together". ${ }^{1}$ Such information is by definition irrelevant, because it doesn't provide BCTs. Similarly, Rosch reports (without comment) that when she carried out learning experiments "on" Dani school kids, who, allegedly, have no words for primary hues at all in their own language, they "would not learn nonsense words", to name the colours of little glossy coloured squares (i.e. Munsell colour chips). ${ }^{2}$ But why wouldn't they want to learn words that named salient, natural, universal, ideal basic categories, of which prototypes or cognitive reference points were presented to them over and over again? Eventually she managed to set up experiments with the Dani in such a way that prototype theory was apparently confirmed, but imagine what sort of conclusions the Dani might have drawn if they would have carried out some sweet potato experiments on Rosch.

\section{@8 holism}

The above and many other examples show that meaning is holistic in Quine's sense of the web of belief, both locally (within one culture) and globally (between cultures); it is plastic and opentextured with respect to meaning use and meaning change. Therefore the "essence" of representation is that it cannot be complete.

Knowing what words mean isn't getting it right in terms of a pre-human ordering of the saliences in the world, or innate hard-wired pre-conceptual prototypes. Getting it right is a matter of grasping the way words are used in social practices.

\footnotetext{
${ }^{1}$ A.R. Luria, Cognitive Development: Its Cultural and Social Foundations, Harvard University Press, 1976:20-29.

2 E.H. Rosch, Linguistic Relativity, in A. Silverstein (ed.) Human Communication: Theoretical Explorations, 1974:114. The problem was solved by using the Dani names for well-known kin-groups as names for the basic colours.
} 
No "rules" for continuance or change exist--reason transcends what it can formally survey. ${ }^{1}$ In this sense human behaviour is both "unpredictable" and "predictable". Or perhaps better: the question of predictability in the philosophy of science sense doesn't make sense. There is both "the same" and "extreme plasticity" in actual interpretative practice.

Ascribing meaning is underdetermined by evolution, adaptivity, or distal stimuli. Information that comes in at the level of perception or desire underdetermines decision. Hence metastrategies humans use for decision making must neglect information at the input level. This is the autonomy of the "metamind", allowing for a plurality of rationalities. ${ }^{2}$

Consider once more the encounter of Cook and the Nuu-chah-nulth. Not only decided Cook to respond to the Nuu-chah-nulth's songs, but also the Nuu-chah-nulth responded to the weird sounds Cook's men were producing. ${ }^{3}$ The event doesn't require a shared prototypical notion of "music" or any other shared prototype, nor does it require explanation in terms of some a priori specifiable necessary precondition of human communication. The Nuu-chah-nulth might have taken the response of Cook to mean that he owned these sounds and that one of the things one does when meeting others is to let them know the songs, stories, dances, objects, etc. one owns-at least that's what they were doing on that particular occasion, or something similar. ${ }^{4}$

Although there is an indefinite number of language games or conceptual schemes (or worlds), they are all based on similar forms of human life, because there are forms of "primitive" or "natural" behaviour which come before the use and understanding of language; it is that on which language games are built. ${ }^{5}$ Interpreting of how we are or could be like in having certain

\footnotetext{
${ }^{1}$ For this sentence and the previous paragraph cf Putnam 1988: 75, 104, 119.

${ }^{2}$ Cf. K. Lehrer, Metamental ascent: Beyond belief and desire, Proceedings American Philosophical Association, 63 (1989) 19-30.

${ }^{3}$ It is quite plausible that "sang in concert in no disagreeable Stile" and "crying out in a mournful tone of voice", in the English and Spanish account respectively, refers to the same sort of thing.

${ }^{4}$ One modern account of the significance of their songs on such occasions is that in doing so they display their privileges coupled with a friendly acknowledgement of the visitor's own prestige.

5 Unser Sprachspiel ist ein Ausbau des primitiven Benehmens [Z545]. "Die gemeinsame menschliche Handlungsweise ist das Bezugssystem, mittels dessen wir uns eine fremde Sprache deuten. (The common behaviour of mankind is the system of references by means of which we interpret an unknown language.)" [PU 206].
} 
practices is therefore what makes (intercultural) communication possible. ${ }^{1}$ However, any attempt to pin down the universal rules underlying human communication gives no more than one way of looking at it. Thus, what is required for knowing how we fit into the world, in having the practices we have, is not explanation under nomological laws, but rather illumination or clarification, by giving examples, showing different ways of looking at the issue, to which there is no end.

To the extent that cognitive science will be successful in providing us with the foundational primitives of human life, it will be by constructing exactly one form of life, a form of life not too much different from that in Brave New World (which isn't too bad after all, or is it?).11

\footnotetext{
${ }^{1}$ Take the concept of personhood. One of the criteria of human personhood is that we are ongoingly interpreters of how we are or could be like others in having certain practices. Such a criterial claim about the nature of persons is not a testable hypothesis about a nomological law. It is, instead, a claim about a point or purpose whose pursuit is evident in various manifold modes of human activity. It is a claim about what we and others in different particular exemplifications aim at, hence a claim that enables us to recognize ourselves in what others do. ["Meine Einstellung zu ihm ist eine Einstellung zur Seele. Ich habe nicht die Meinung dasz er eine Seele hat. (My attitude towards him is an attitude towards a soul. I am not of the opinion that he has a soul.)" Wittgenstein, PI, II, iv]
} 